\title{
AUTOEFICACIA GENERAL, ANSIEDAD PRECOMPETITIVA Y SENSACIÓN DE FLUIR EN JUGADORES (AS) DE BALONMANO DE LA SELECCIÓN NACIONAL DE COSTA RICA
}

\author{
Juan Carlos Gutiérrez Vargas, Randall Gutiérrez Vargas, Pedro \\ Ureña Bonilla \\ Escuela de Ciencias del Movimiento Humano y Calidad de Vida. \\ Facultad de Ciencias de la Salud, Universidad Nacional de Costa \\ Rica. \\ juan.gutierrez.vargas@una.cr
}

RESUMEN

El propósito de este estudio fue describir y relacionar los niveles de autoeficacia, ansiedad precompetitiva y sensación de fluir en jugadores de balonmano de la selección nacional masculina y femenina de Costa Rica durante el Torneo Internacional Feria de la Flores (Colombia 2012). En el estudio participaron 28 jugadores (14 hombres y 14 mujeres). Se aplicó la escalas de autoeficacia general, sensación de fluir y ansiedad competitiva. El valor promedio en relación con la autoeficacia fue alto $(8,40)$. En cuanto a la sensación de fluir, los valores promedio oscilaron entre 3,41 (experiencia autotélica) y 5,78 (sensación de control). La ansiedad somática fue la más baja (hombres $=1,59 /$ mujeres $=1,98)$ y la autoconfianza la más alta (hombres $=2,99 /$ mujeres $=2,70$ ). No se registró cambios significativos a lo largo de los juegos en relación con la sensación de fluir. La ansiedad experimentada por los hombres fue significativamente menor a la de las mujeres, y los niveles de autoconfianza fueron superiores en los hombres que en las mujeres. No se registró correlaciones importantes entre autoeficacia y sensación de fluir; sin embargo, la ansiedad somática mostró correlaciones significativas con algunas dimensiones de la sensación de fluir. Los datos registrados evidencian la nece sidad del trabajo psicológico, para el logro de estados psicofísicos que favorezcan el logro de rendimientos máximos.

PALABRAS CLAVE: experiencia de fluir, auto-eficacia, ansiedad, autoconfianza.

ABSTRACT

The purpose of this study was to describe and analyze the relationship between self-efficacy, pre-competitive anxiety and flow feeling in male and female handball team players from the Costa Rica national teams. Participants were 28 players (14 male and 14 female) from both teams. The scales of general self-efficacy, flow feeling and competitive anxiety were used to collect data. The average score in relation to self-efficacy was high $(>8.40)$. Regarding flow sensation, the average scores were range from 3.41 (autotelic experience) to 5,78 (control sense). Somatic anxiety was the lowest in men $=1.59$ and women female $=1.98$, and self-confidence was the highest score in men $=2.99$ and women $=2.70$ respectively. No significant changes were observed throughout the game in relation to the flow feeling. The anxiety reported by men was significantly lower than women, and the self-confidence levels were higher in men than in women. No significant correlation was found between self efficacy and sense of flow. Somatic anxiety showed significant correlations with some dimensions of the flow feeling. In conclusion, these data showed that there is a need to incorporate psychological interventions to ensure that athletes can reach optimal psychophysical states in order to perform better.

KEY WORDS: flow feeling, self efficacy, anxiety, self confidence 


\section{INTRODUCCIÓN}

Numerosos autores señalan la relación entre factores psicológicos y ejecución deportiva (Dosil, 2004; Sherman y Poczwardowski, 2005; Boillos, 2006; Weinberg y Gould, 2007). La autoeficacia (Feltz, Short y Sullivan, 2008; Myers y Feltz, 2007), la ansiedad precompetitiva (Ranglin y Hannin, 2000) y la sensación de fluir (Fortier y Kowal, 2007; Jackson, 2000) son algunos de los constructos que con frecuencia se reportan vinculados al rendimiento de los deportistas. La autoeficacia es un constructo acuñado por Bandura (1986) y se entiende como la convicción que tiene el individuo en relación con su capacidad para hacerle frente con éxito a las exigencias que presenta la vida cotidiana (Ceja y Navarro, 2009; Bandura, 1986). Se trata de una sensación de involucramiento total (Nacke y Lindley, 2008), que se vincula con una motivación intrínseca superior (Hong, Chiu, Shih y Lin, 2012), por lo que su efecto sobre el rendimiento deportivo puede ser muy positivo (Feltz et al., 2008; Moritz et al., 2000). Las personas con altos niveles de eficacia son proactivos en la regulación de sus cogniciones, motivaciones y emociones, lo que le da ventaja para enfrentar los retos que se les presenten. En contraste, las personas con baja eficacia evitan metas difíciles, se preocupan demasiado por las cosas, se esfuerzan menos y se rinden ante el fracaso (Feltz et al., 2008). Es importante señalar que, las creencias relacionadas con la eficacia son específicas de diferentes dominios, es decir, la naturaleza de la situación juega un papel central en el desarrollo de las creencias de eficacia. Sin embargo, una cosa es poseer una serie de destrezas y otra es tener la confianza para hacer uso funcional de esas destrezas en el curso de una acción o serie de acciones.

La autoeficacia está determinada fundamentalmente por las experiencias de maestría "mastery experience" que el individuo haya vivido a lo largo de su desarrollo ontogenético (Breso, Schaufeli y Salanova, 2011); es decir, de vivencias en donde la persona haya experimentado el dominio de una o varias destrezas en particular. Las expectativas de maestría inciden sobre el rendimiento pero también, los buenos logros inciden sobre las mismas, es decir, altos niveles de eficacia tienden a aumentar el rendimiento, y a la inversa, los buenos rendimientos tienden a aumentar las creencias de eficacia (Feltz et al., 2008). Desde esta perspectiva, niveles altos de autoeficacia se correlacionan significativa y positivamente con niveles apropiados de ansiedad, estrés y fatiga (Breso et al., 2011). Es decir, la eficacia percibida influencia los niveles de estrés y ansiedad por medio de las creencias de la persona en relación con el control de sus acciones, pensamientos y afectos (Feltz et al., 2008).

En el campo de la competencia deportiva se distingue diferentes tipos de autoeficacia dentro de las que se mencionan eficacia paliativa, colectiva, competitiva, afrontamiento, aprendizaje, ejecución, preparatoria, auto-reguladora y de tarea. De hecho las creencias de eficacia son los determinantes primarios de los niveles motivacionales de las personas cuando se procura una meta. De manera que, las personas con altos niveles de eficacia comparados con aquellos que tienen menores niveles, tienden a escoger metas retadoras, 
perciben los esfuerzos que hacen como menores y tienen una mayor persistencia en procura de las metas que desean (Feltz et al., 2008).

Por otro lado, la ansiedad es un constructo que ha sido extensamente estudiado en el campo de la psicológica deportiva (León, Fuentes y Calvo, 2011). Se refiere a estados emocionales inapropiados para el rendimiento deportivo, caracterizados por nerviosismo y preocupación (Weinberg y Gould, 2007; Hanin, 2007). En general, se diferencia entre ansiedad cognitiva y somática (Ramis, Torregosa, Viladrich y Cruz, 2010). La ansiedad cognitiva se vincula a las preocupaciones que caracterizan el estilo de pensamiento del deportista, mientras que la ansiedad somática refleja el correlato orgánico de ese estado emocional (Hernández, Olmedilla y Ortega, 2008).

La ansiedad puede asociarse con malos rendimientos en la medida en que incida negativamente sobre los procesos atencionales, la toma de decisiones y el control emocional (Grossbard, Smith, Small y Cumming, 2009; Thomas, Maynard y Hanton, 2004; Márquez, 2004). Estados emocionales negativos como la ansiedad, influencian negativamente el juzgamiento que hace el deportista de su eficacia; mientras que estados afectivos positivos, como la alegría, mejoran la forma en que la persona juzga su propio rendimiento. Por lo tanto, se puede hablar de una relación inversamente proporcional entre ansiedad y autoeficacia (Cartoni, Mongote y Zelli, 2005).

Asimismo, cuando los valores de ansiedad cognitiva y somática caen dentro de la zona individual de funcionamiento óptimo (Ranglin y Hanin, 2000), el rendimiento del deportista tiende a ser máximo (Cervantes, Rodas y Capdevila, 2009), por lo que puede hablarse de un rango donde los niveles de ansiedad se correlacionan con ejecuciones óptimas. En este contexto, la autoconfianza ha sido considerada un factor mediador de la ansiedad (Hernández, Olmedilla y Ortega, 2008). De manera que, deportistas con altos grados de autoconfianza tienden a manejar mejor dicho estado emocional (Feltz, et al., 2008; Covassin y Pero, 2004). De hecho Jaenes, Peñaloza, Navarrete y Bohórquez (2012) se refieren a la ansiedad somática, cognitiva y autoconfianza, como la triada de factores decisivos en el correlato emocional precompetitivo. En este sentido, se ha reportado relaciones significativas y positivas entre autoconfianza y rendimiento (Coudevylle, Ginis y Famose, 2008; Sáenz-López, Jiménez, Ibañez, Sierra y Sánchez, 2005). No cabe duda que la autoconfianza es un recurso de mediación fundamental entre el deportista y los síntomas que genera la preocupación y la subsecuente interpretación que éste hace de la misma (Mellaliu, Neil y Hanton, 2006).

Desde el punto de vista de la teoría cognitiva, la ansiedad es considerada un efecto derivado de un bajo nivel de eficacia. En relación con el sexo, la literatura reporta que, las mujeres comparadas con los hombres presentan niveles más altos de ansiedad cognitiva y somática, asimismo, muestran valores inferiores en autoconfianza (Martens, Burton, Vealey, Bump y Smith, 1990).

Finalmente, la sensación de fluir corresponde a la tradición creada por el enfoque positivo de la psicología (Seligman y Csikszentmihalyi, 2000). De acuerdo con Csikszentmihalyi 
(1992), la experiencia de fluir se caracteriza por una serie de sensaciones determinantes para el rendimiento del deportista (Jackson, 2000; García, Jiménez, Santos, Reina y Cervello, 2008; Nacke y Lindley, 2008; Hodge, Lonsdale y Jackson, 2009; Yamamoto y Avery, 2009; Bakker, Oerlemans, Demerouti, Bruins y Karamat, 2011): (A) el balance entre destrezas y reto, osea el deportista percibe que sus habilidades son suficientes para enfrentar con éxito los desafíos que se le presentan. (B) La completa absorción en la actividad (fusión en la acción), que implica que el deportista está concentrado en la tarea y la ejecución de la misma transcurre automáticamente, evitando pensamientos que entorpecen. (C) Claridad de metas, lo que le permite al deportista optimizar su rendimiento, especialmente por la focalización de la atención en la tarea. (D) Realimentación directa y clara, aspecto esencial para saber si se está haciendo las cosas correctamente. (E) Concentración en la tarea que se está ejecutando, característica fundamental para lograr rendimientos óptimos. (F) Sentido de control, esta dimensión se refiere a la sensación de control y maestría en relación con la tarea que se está realizando. (G) Pérdida de la autoconciencia o inhibición, la cual refleja la capacidad que tiene el deportista para evitar preocupaciones en relación con la propia destreza. (H) Distorsión del sentido del tiempo, la que se caracteriza por que la sensación del tiempo se ve alterada en interés propio, estimulando una actitud positiva y favoreciendo una mejor ejecución. (I) Experiencia autotélica, la cual hace referencia a la satisfacción intrínseca producida por la tarea. En esencia, la sensación de fluir es un fenómeno multifacético que incluye muchas características y que se asocia con una experiencia afectiva positiva (Schüler y Engenser, 2009; Fortier y Kowal, 2007; Jackson, 2000).

Podría decirse que la sensación de fluir es el resultado de una gran motivación intrínseca (Fortier y Kowel, 2007) y se relaciona de manera estrecha con variables motivacionales como por ejemplo la competencia percibida (Martin y Cutler, 2002). La sensación de fluir se relaciona positivamente con el rendimiento deportivo por dos razones principales: la primera, porque estimula el rendimiento por sí mismo y la segunda, porque los individuos que experimentan esa sensación están más motivados para llevar a cabo actividades con la intención de experimentar nuevamente ese estado psicofísico, por lo que se pondrán metas retadoras (Bakker et al., 2011).

El estado del conocimiento alrededor de esta problemática sugiere relaciones estrechas entre autoeficacia, ansiedad y sensación de fluir. En general los deportistas con una mejor autoeficacia, reportan menos ansiedad precompetencia (Breso et al., 2011) y mayores valores en sensación de fluir. En el marco de estos antecedentes, el propósito de este estudio fue describir y relacionar los niveles de autoeficacia general, ansiedad precompetitiva y sensación de fluir en jugadores (as) de las selecciones de balonmano de Costa Rica, durante el Torneo Internacional de la "Feria de las Flores" llevado a cabo en agosto del 2012 en Colombia. Es válido mencionar que este estudio representa un primer intento por introducir la psicología del deporte en el balonmano costarricense, a partir de datos descriptivos y derivaciones correlativas, lo que agrega valor indiscutible a esta experiencia 


\section{METODOLOGIA}

Sujetos. Se contó con la participación de los jugadores (as) de la selección de balonmano de Costa Rica $(n=28)$. El equipo masculino estuvo integrado por 14 jugadores con una edad promedio de 27,57 $\pm 5,09$ años, y el femenino por 14 jugadoras con una edad promedio de $22,84 \pm 3,7$ años.

Instrumentos. Se aplicó la escala de autoeficacia general propuesta por Espada, González, Orgilés, Carballo \& Piqueras (2012); la que está compuesta por 10 ítems, que son evaluados mediante una escala likert de 10 opciones, para identificar el nivel de acuerdo en relación con cada ítem, donde 1 corresponde a totalmente en desacuerdo y 10 a totalmente de acuerdo. Puntajes superiores son indicadores de una mejor autoeficacia. Se le reporta una consistencia interna correspondiente a un alfa de Cronbach de 0.89 , así como también buena estabilidad temporal y validez. La escala CSAI-2 de ansiedad competitiva de Martens, Burton, Vealey, Bump \& Smith (1982), traducción realizada por 2 personas bilingües (español - inglés) en procura de mantener el sentido original de los ítems, y posteriormente confrontada con el criterio de los autores. Este instrumento consta de 27 ítems distribuidos en 3 categorías: ansiedad cognitiva, ansiedad somática y autoconfianza. Valores superiores en cada factor son indicadores de mayores niveles de ansiedad cognitiva, somática, pero también de mayor autoconfianza (Martens et al., 1982). Para este instrumento se reportan coeficientes de consistencia interna superiores a 0.70 en cada subescala (Lane, Sewell, Terry, Bartram, y Nesti, 1999) y utiliza un formato de respuesta tipo likert de 4 opciones, donde 1 corresponde a nada y 4 a mucho.

Se utilizó también la escala de fluir, en su versión española propuesta por Calvo, Jiménez, Santos-Rosa, Reina y Cervello (2008), la cual consta de 36 ítems distribuidos en 9 factores: balance entre nivel de destreza y reto (BDR), concentración en la tarea (CT), realimentación directa (RD), distorsión del sentido del tiempo (DT), sentido de control (SC), experiencia autotélica (EA), claridad de metas (CM), funcionamiento automático (FA) y pérdida de la autoconciencia (PA). El formato de respuesta es tipo likert de 7 puntos, donde 1 corresponde a totalmente en desacuerdo y 7 a totalmente de acuerdo. Valores superiores según la escala de valoración respectiva son reflejo de una mejor disposición para vivir la experiencia de fluir (Calvo et al., 2008). Para esta escala se reporta coeficientes de consistencia interna (alfa de Cronbach) superiores a 0.70 en cada sub escala (Calvo et al., 2008).

Asimismo, se utilizaron dos ítems elaborados por los investigadores para valorar la autopercepción de los participantes en relación con la condición física y técnica actual, para lo cual se utilizó una escala tipo likert que iba de $0-10$, donde 0 corresponde a la peor valoración y 10 a la mejor valoración.

Procedimiento. Todas las mediciones se realizaron durante el Torneo Internacional "Feria de las Flores" celebrado en Colombia en agosto del 2012. La selección femenina jugó 5 partidos y la masculina 7. La escala de autoeficacia general se aplicó un día antes de salir hacia el citado torneo, asimismo, antes y después de cada partido tanto de la selección 
femenina como masculina, se aplicó el test de ansiedad precompetitiva y la escala de fluir respectivamente. La ansiedad precompetitiva fue medida 1 hora antes de cada partido y se aplicó antes de todos los juegos realizados tanto por la selección masculina como femenina. El cuestionario de autoeficacia general se aplicó antes de partir al torneo internacional supra-referido. La escala de fluir adaptada se administró 30 minutos después de finalizado cada partido. La participación de los (as) jugadores (as) fue voluntaria y a todos ellos se les explicó el propósito de la investigación que se estaba realizando.

Análisis estadístico. Se calculó estadísticas descriptivas (promedios y desviaciones típicas), correlaciones producto momento de Pearson, contraste de promedios mediante tstudent para las comparaciones según sexo y análisis de varianza con medidas repetidas y con ajuste Bonferroni para el seguimiento de los factores estudiados a lo largo de los juegos realizados por la selección femenina y masculina. El nivel de significancia utilizado para todos los contrastes fue de 0,05 .

\section{RESULTADOS}

La apreciación de la condición física por parte de los hombres arrojó valores promedio de $7,33 \pm 1,33$ y para las mujeres de 7,07 $\pm 1,63$. No se encontró diferencias significativas entre ambos grupos $(\mathrm{p}>0,05)$. En cuanto a la valoración de la condición técnica, el promedio para los hombres fue de 7,64 $\pm 1,08$ y para las mujeres de $6,71 \pm 1,63$. No se registró diferencias importantes entre ambos grupos $(p>0,05)$. Asimismo, la autoeficacia general de los hombres mostró un promedio de 8,41 $\pm 0,70$ y para las mujeres de 8,59 \pm 0,88 , sin diferencias estadísticas entre ambos grupos $(\mathrm{p}>0,05)$.

\section{Tabla 1}

VALORES PROMEDIO Y DESVIACIONES TÍPICAS RELATIVAS A LA SENSACIÓN DE FLUIR EN MUJERES DESPUÉS DE CADA JUEGO

\begin{tabular}{lrrrrr}
\hline Fluir & $\mathbf{J - 1}$ & $\mathbf{J - 2}$ & $\mathbf{J - 3}$ & $\mathbf{J - 4}$ & $\mathbf{J - 5}$ \\
\hline BDR & $5,38 \pm 1,04$ & $5,57 \pm 1,08$ & $4,42 \pm 1,45$ & $5,57 \pm 0,93$ & $5,28 \pm 0,91$ \\
FA & $5,46 \pm 1,19$ & $5,07 \pm 1,14$ & $4,92 \pm 1,32$ & $5,14 \pm 0,77$ & $4,92 \pm 1,54$ \\
CM & $5,23 \pm 1,01$ & $4,92 \pm 0,99$ & $4,78 \pm 1,62$ & $5,14 \pm 0,86$ & $4,64 \pm 1,49$ \\
RD & $4,23 \pm 2,08$ & $3,85 \pm 2,21$ & $3,14 \pm 1,65$ & $4,57 \pm 2,10$ & $4,00 \pm 1,79$ \\
CT & $5,15 \pm 1,28$ & $5,28 \pm 1,06$ & $5,07 \pm 1,32$ & $5,00 \pm 0,96$ & $4,57 \pm 1,69$ \\
SC & $6,61 \pm 0,66$ & $6,42 \pm 0,64$ & $5,92 \pm 1,38$ & $5,28 \pm 1,63$ & $5,14 \pm 1,83$ \\
PA & $6,07 \pm 1,11$ & $6,07 \pm 0,82$ & $6,07 \pm 0,91$ & $5,42 \pm 1,60$ & $5,57 \pm 1,65$ \\
DT & $4,07 \pm 2,06$ & $3,64 \pm 1,33$ & $3,57 \pm 1,78$ & $3,78 \pm 1,84$ & $3,78 \pm 1,71$ \\
EA & $3,61 \pm 2,32$ & $3,21 \pm 2,08$ & $4,07 \pm 2,20$ & $3,42 \pm 1,98$ & $3,00 \pm 1,51$ \\
\hline
\end{tabular}

$\mathrm{BDR}=$ Balance entre destreza y reto, $\mathrm{FA}=$ Funcionamiento automático, $\mathrm{CM}=$ Claridad de metas, $\mathrm{RD}=$ Retroalimentación directa, $\mathrm{CT}=$ Concentración en la tarea, $\mathrm{SC}=$ Sentido de control, $\mathrm{PA}=$ Pérdida de autoconciencia, $\mathrm{DT}=$ Distorsión del sentido del tiempo, $\mathrm{EA}=$ Experiencia autotélica/ $\mathrm{J}=$ juego. 
De acuerdo con la lectura de la tabla 1, los valores promedio más bajos corresponden a experiencia autotélica, distorsión del sentido del tiempo y realimentación directa. Como contraste los valores promedio superiores fueron registrados en los factores: sentido de control y pérdida de autoconciencia. Análisis de varianza para medidas repetidas no encontró diferencias importantes relacionadas con la sensación de fluir entre juegos $(\mathrm{p}>$ $0,05)$.

Tabla 2

VALORES PROMEDIO Y DESVIACIONES TÍPICAS RELATIVAS A LA SENSACIÓN DE FLUIR EN HOMBRES DESPUÉS DE CADA JUEGO

\begin{tabular}{lccccccc}
\hline \multicolumn{1}{c}{ Fluir } & $\mathbf{J - 1}$ & $\mathbf{J - 2}$ & $\mathbf{J - 3}$ & $\mathbf{J}-\mathbf{4}$ & $\mathbf{J - 5}$ & $\mathbf{J - 6}$ & $\mathbf{J - 7}$ \\
\hline BDR & $5,50 \pm 1,04$ & $5,85 \pm 0,77$ & $6,28 \pm 0,72$ & $5,63 \pm 0,92$ & $6,00 \pm 1,20$ & $5,76 \pm 1,48$ & $5,91 \pm 1,56$ \\
FA & $5,42 \pm 0,93$ & $5,69 \pm 0,63$ & $5,71 \pm 1,13$ & $5,54 \pm 0,93$ & $5,83 \pm 1,46$ & $5,46 \pm 1,50$ & $6,08 \pm 0,99$ \\
CM & $5,07 \pm 0,99$ & $5,07 \pm 0,99$ & $4,85 \pm 1,87$ & $5,20 \pm 0,91$ & $5,50 \pm 1,38$ & $5,07 \pm 1,70$ & $5,83 \pm 1,26$ \\
RD & $4,57 \pm 2,17$ & $5,00 \pm 1,61$ & $4,28 \pm 2,05$ & $4,36 \pm 1,96$ & $4,58 \pm 2,19$ & $4,38 \pm 2,39$ & $4,91 \pm 2,23$ \\
CT & $5,21 \pm 1,12$ & $5,42 \pm 1,22$ & $5,07 \pm 1,63$ & $5,18 \pm 0,87$ & $5,50 \pm 1,24$ & $5,76 \pm 1,30$ & $5,50 \pm 1,31$ \\
SC & $6,28 \pm 0,91$ & $6,35 \pm 0,63$ & $6,00 \pm 1,46$ & $6,18 \pm 0,75$ & $6,16 \pm 1,02$ & $6,25 \pm 1,13$ & $6,50 \pm 0,63$ \\
PA & $6,50 \pm 0,65$ & $6,28 \pm 0,72$ & $5,92 \pm 1,63$ & $6,27 \pm 0,78$ & $5,83 \pm 1,64$ & $5,92 \pm 1,75$ & $6.50 \pm 0,52$ \\
DT & $3,71 \pm 1,58$ & $3,92 \pm 1,63$ & $4,14 \pm 1,70$ & $4,72 \pm 1,55$ & $3,16 \pm 1,58$ & $4,15 \pm 1,77$ & $4,00 \pm 1,75$ \\
EA & $4,14 \pm 1,74$ & $4,57 \pm 1,65$ & $3,71 \pm 1,72$ & $4,27 \pm 1,84$ & $3,33 \pm 1,87$ & $4,23 \pm 1,83$ & $4,08 \pm 2,10$ \\
\hline BD & &
\end{tabular}

$\mathrm{BDR}=$ Balance entre destreza y reto, $\mathrm{FA}=$ Funcionamiento automático, $\mathrm{CM}=$ Claridad de metas, $\mathrm{RD}=\mathrm{Retroalimentación} \mathrm{directa,} \mathrm{CT}=$ Concentración en la tarea, $\mathrm{SC}=$ Sentido de control, $\mathrm{PA}=$ Pérdida de autoconciencia, $\mathrm{DT}=$ Distorsión del sentido del tiempo, $\mathrm{AE}=$ Experiencia autotélica / $\mathrm{J}=$ juego.

Los valores contenidos en la Tabla 2, muestran los promedios más bajos en las categorías: distorsión del tiempo, experiencia autotélica y realimentación directa. Como contraste, los promedios más altos se registran en los factores, pérdida de autoconciencia, sentido de control y balance entre destreza y reto. No se registró diferencias significativas entre los valores promedio según juego $(\mathrm{p}>0,05)$.

Tabla 3

VALORES PROMEDIO Y DESVIACIONES TÍPICAS RELATIVAS A LA ANSIEDAD PRECOMPETITIVA EN MUJERES ANTES DE CADA JUEGO

\begin{tabular}{lccccc}
\hline Ansiedad & $\mathbf{J - 1}$ & $\mathbf{J - 2}$ & $\mathbf{J - 3}$ & $\mathbf{J - 4}$ & $\mathbf{J - 5}$ \\
\hline Cognitiva & $2,65 \pm 0,63$ & $2,63 \pm 0,47$ & $2,59 \pm 0,59$ & $2,59 \pm 0,69$ & $2,51 \pm 0,60$ \\
Somática & $2,25 \pm 0,53 \mathbf{a}$ & $1,97 \pm 0,57$ & $2,03 \pm 0,51$ & $1,80 \pm 0,49 \mathbf{b}$ & $1,85 \pm 0,63$ \\
Autoconfianza & $2,74 \pm 0,57$ & $2,87 \pm 0,39 \mathbf{a}$ & $2,03 \pm 0,51 \mathbf{b}$ & $2,69 \pm 0,50$ & $2,73 \pm 0,39$ \\
\hline Letras diferentes evidencian diferencias entre grupos $(\mathrm{p}<0,05)$ & &
\end{tabular}


Como se observa en la Tabla 3, los valores promedio más altos corresponden al factor autoconfianza. Un segundo rango en importancia según los valores promedio registrados, lo ocupa la vivencia de ansiedad cognitiva. Análisis de varianza para medidas repetidas mostró diferencias significativas entre juegos en relación con los factores ansiedad somática y autoconfianza. En el primero de los casos, se registra diferencias significativas entre el J-1 y J-4. Los valores más bajos relativos a la ansiedad somática se registraron en el juego 4. En cuanto a la autoconfianza, las diferencias se dan entre los juegos J-2 y J-3. Los valores más altos relativos a la autoconfianza se registraron en el J-2.

Tabla 4

VALORES PROMEDIO Y DESVIACIONES TÍPICAS RELATIVAS A LA ANSIEDAD PRECOMPETITIVA EN HOMBRES ANTES DE CADA JUEGO

\begin{tabular}{lccccccc}
\hline Ansiedad & $\mathbf{J - 1}$ & $\mathbf{J - 2}$ & $\mathbf{J - 3}$ & $\mathbf{J}-\mathbf{4}$ & $\mathbf{J - 5}$ & $\mathbf{J - 6}$ & J-7 \\
\hline Cognitiva & $1,92 \pm 0,30$ & $1,80 \pm 0,44$ & $2,11 \pm 0,49$ & $2,00 \pm 0,52$ & $1,82 \pm 0,68$ & $2,15 \pm 1,03$ & $1,83 \pm 0,52$ \\
Somática & $1,76 \pm 0,33$ & $1,64 \pm 0,56$ & $1,57 \pm 0,31$ & $1,66 \pm 0,41$ & $1,47 \pm 0,30$ & $1,92 \pm 1,19$ & $1,55 \pm 0,44$ \\
Autoconfianza & $3,05 \pm 0,50$ & $3,23 \pm 0,58$ & $2,96 \pm 0,64$ & $2,92 \pm 0,64$ & $3,38 \pm 0,38$ & $3,22 \pm 0,85$ & $3,12 \pm 0,60$ \\
\hline
\end{tabular}

De acuerdo con los valores contenidos en la tabla 4, los valores promedio más altos corresponden al factor autoconfianza a lo largo de los 7 juegos, seguido por los valores pertenecientes al factor ansiedad cognitiva. Análisis de varianza para medidas repetidas no mostró diferencias importantes entre juegos en relación con los factores evaluados $(\mathrm{p}>$ $0,05)$.

Tabla 5

VALORES PROMEDIO GLOBALES Y DESVIACIONES TÍPICAS RELATIVAS A LA SENSACIÓN DE FLUIR SEGÚN SEXO

\begin{tabular}{lccc}
\hline Fluir general & Masculino & Femenino & Sig. \\
\hline BDR & $5,37 \pm 0,88$ & $5,17 \pm 0,47$ & 0,45 \\
FA & $5,15 \pm 0,91$ & $5,02 \pm 0,75$ & 0,69 \\
CM & $4,73 \pm 0,91$ & $4,87 \pm 0,64$ & 0,65 \\
RD & $4,21 \pm 1,55$ & $3,90 \pm 1,39$ & 0,57 \\
CT & $4,93 \pm 0,73$ & $4,94 \pm 0,64$ & 0,98 \\
SC & $5,67 \pm 0,75$ & $5,78 \pm 0,77$ & 0,70 \\
PA & $5,67 \pm 0,81$ & $5,75 \pm 0,76$ & 0,78 \\
DT & $3,64 \pm 1,10$ & $3,71 \pm 1,05$ & 0,86 \\
EA & $3,72 \pm 1,32$ & $3,41 \pm 1,29$ & 0,53
\end{tabular}

$\mathrm{BDR}=$ Balance entre destreza y reto, $\mathrm{FA}=$ Funcionamiento automático, $\mathrm{CM}=$ Claridad de metas, $\mathrm{RD}=$ Retroalimentación directa, $\mathrm{CT}=$ Concentración en la tarea, $\mathrm{SC}=$ Sentido de control, $\mathrm{PA}=$ Pérdida de autoconciencia, $\mathrm{DT}=$ Distorsión del sentido del tiempo, $\mathrm{EA}=$ Experiencia autotélica 
La lectura de la Tabla 5, permite evidenciar que no existen diferencias significativas en cuanto a la sensación de fluir según el sexo. Los valores promedio generales más bajos relativos a la sensación de fluir corresponden a los factores: distorsión del tiempo, experiencia autotélica y realimentación del rendimiento. En contraste, los promedios más altos están en los factores, pérdida de la autoconciencia, sentido de control y balance entre destreza y reto.

Tabla 6

VALORES PROMEDIO GLOBALES Y DESVIACIONES TÍPICAS RELATIVAS A LA ANSIEDAD PRECOMPETITIVA GENERAL SEGÚN SEXO

\begin{tabular}{lccc}
\hline Ansiedad & Masculino & Femenino & Sig. \\
\hline Cognitiva & $1,87 \pm 0,45$ & $2,60 \pm 0,47$ & 0,00 \\
Somática & $1,59 \pm 0,33$ & $1,98 \pm 0,45$ & 0,01 \\
Autoconfianza & $2,99 \pm 0,35$ & $2,70 \pm 0,36$ & 0,04 \\
\hline
\end{tabular}

De acuerdo con la información contenida en la Tabla 6, hay diferencias importantes en cuanto a la vivencia de ansiedad precompetitiva según el sexo. Los hombres comparados con las mujeres, muestran valores más bajos en ansiedad cognitiva y somática, así como también, evidencian un puntaje superior en autoconfianza.

Se realizó análisis de correlación a partir de los valores globales tanto en la escala de fluir como en la escala de ansiedad precompetitiva. No se encontró correlaciones significativas entre las dimensiones de la escala de fluir y el valor promedio general de la autoeficacia ( $\mathrm{p}$ $>0,05)$. Sin embargo, la ansiedad somática global se correlacionó significativamente con el ítem "distorsión del sentido del tiempo" $(\mathrm{r}=0,40 ; \mathrm{p}=0,03)$. Por otro lado, la autoconfianza mostró relaciones significativas con los ítems "balance entre destreza y reto" $(\mathrm{r}=0,49 ; \mathrm{p}=0,008)$, "funcionamiento automático" $(\mathrm{r}=0,38 ; \mathrm{p}=0,04)$ y "pérdida de autoconciencia" ( $\mathrm{r}=0,38 ; \mathrm{p}=0,04)$. Asimismo, la valoración subjetiva de la condición física se correlacionó significativamente con la condición técnica $(r=0,39 ; p=0,03)$, con la autoeficacia $(r=0,37 ; p=0,04)$ y con experiencia autotélica $(0,42 ; p=0,02)$. No se registró correlaciones significativas entre condición técnica y la sensación de fluir global $(\mathrm{p}>0,05)$; tampoco entre autoeficacia general y las dimensiones correspondientes a la sensación de fluir $(\mathrm{p}>0,05)$.

\section{DISCUSIÓN}

En relación con la autoeficacia general, los resultados encontrados evidencian a un grupo de deportistas con una alta convicción (de acuerdo a la escala de respuesta) en cuanto a su capacidad para hacerle frente a los retos que la vida les presenta, lo que podría asociarse 
con un nivel elevado de motivación intrínseca. En estos deportistas podría esperarse un involucramiento intenso con la tarea que realizan (Nacke y Lindley, 2008) y una alta motivación interna (Hong et al., 2012). Por otro lado, se puede suponer en estos (as) deportistas experiencias positivas en situaciones generales de la vida diaria que fortalecen la autoeficacia (Breso et al., 2010). Sin embargo, la autoeficacia no se asoció de manera significativa con las dimensiones de la sensación de fluir. Esto podría indicar que tener gran convicción para enfrentar los retos de la vida diaria, no necesariamente es una condición fundamental, para que los deportistas experimenten la sensación de fluir en competencia deportiva. Podría argumentarse que la competencia deportiva tiene características muy particulares, por lo que tendría que acuñarse un concepto diferenciado de autoeficacia, es decir específico para cada deporte (Hernández, Velásquez, Martínez, Garoz y Tejero, 2011; Feltz et al., 2008). Aunque la autoeficacia general, sí se vinculó de manera significativa a la percepción subjetiva de la condición física que tienen los deportistas; lo cual indica que sentirse bien físicamente es importante para tener esa convicción general en relación con la capacidad para resolver dificultades del diario vivir. De manera que, la sensación de una buena condición física es fundamental para sentirse en capacidad de resolver las eventualidades que la vida presenta.

Por otro lado, en cuanto a la sensación de fluir los valores promedio registrados evidenciaron un perfil promedio alto en el $44 \%$ de las dimensiones de este constructo. Sin embargo, en la mayoría de los factores que caracterizan la sensación de fluir se registró valores relativamente bajos. El valor más bajo se da en relación con la experiencia autotélica, lo que podría evidenciar el nivel reducido de satisfacción intrínseca que experimentaron los deportistas encuestados en relación la competencia vivida a lo largo del torneo. Esta situación no solo puede afectar el nivel de disfrute de los deportistas sino que también podría incidir negativamente en su rendimiento (Jackson, 2000; Fortier y Kowal, 2007). Similar comportamiento se observa en relación con el factor distorsión del tiempo.

Los valores promedio registrados parecen evidenciar que los (las) deportistas encuestados (as) no estuvieron suficientemente compenetrados con el juego, como para experimentar alteraciones en la percepción del tiempo en beneficio propio, por lo que difícilmente podría esperarse una actitud positiva y un nivel motivacional óptimo. En este sentido, por ejemplo, percibir que el juego transcurrió velozmente, podría significar una gran concentración del deportista, lo que se interpreta como beneficio para el deportista (Abernethy, Maxuell, Masters, Van Der Kamp y Jackson, 2007). Otro factor que mostró valores relativamente bajos es la realimentación directa, lo cual podría indicar que los deportistas que participaron en este torneo, no tienen la costumbre de realimentar positiva y permanentemente su desempeño en la competencia.

Aunque paradójicamente, estos deportistas reportaron valores relativamente altos en cuanto a sensación de control y maestría en el desempeño, así como valores relativamente bajos relativos a la preocupación por la propia destreza. En este sentido es importante señalar que, el proceso de realimentación es una fuente directa de percepción de competencia (Hein y Koka, 2007) y es fundamental para el deportista en la medida en que puede contribuir con 
los procesos de autorregulación necesarios para realizar ajustes psicomotrices y cognitivoemocionales en función de un mejor rendimiento (Weinberg y Gould, 2007).

A lo largo de todos los juegos, se mantuvo un perfil similar relativo a la ansiedad somática. En el caso de los hombres no hubo diferencias inter-juegos, es decir, la respuesta orgánica del estado emocional denominado ansiedad fue similar. Sin embargo, en el caso de las mujeres, la ansiedad somática del primer juego fue la mayor y difiere significativamente de la experimentada en el juego número 4, no así en los demás juegos. Esto se puede explicar por cuanto los primeros juegos en torneos de esta naturaleza suelen generar respuestas orgánicas ansiógenas, que podrían atentar contra un buen rendimiento (Hanin, 2007; Ranglin y Hanin, 2000). Asimismo, para el caso de las mujeres, el menor nivel de autoconfianza se registró en el juego 3. En contraste, los hombres mostraron valores promedio similares en cuanto a los factores de la ansiedad a lo largo de todos los juegos. La autoconfianza alcanzó los mayores puntajes, lo que podría considerase como evidencia de que los deportistas encuestados poseen buena seguridad en sí mismos y por lo tanto buena capacidad para controlar su ansiedad. Por otro lado, los hombres comparados con las mujeres evidenciaron un estilo de pensamiento menos ansiogénico, una menor respuesta somática ante la ansiedad y un nivel superior de autoconfianza. Estos datos concuerdan con Martens et al (1990), quienes refieren que las mujeres puntúan más alto que los hombres en ansiedad somática - cognitiva, y tienen además menor autoconfianza.

En términos de asociación, la reacción somática ante la ansiedad se vinculó de manera positiva y significativa con la sensación de distorsión del tiempo, lo que indica que un nivel de ansiedad somática específico podría ser importante, para experimentar alteraciones en la sensación del tiempo y obtener mejores rendimientos. En este contexto el modelo de zonas de óptimo funcionamiento individual propuesto por Ranglin y Hannin (2000), pareciera ser de utilidad explicativa de este fenómeno. Es decir, no es la absoluta ausencia de una respuesta orgánica ansiógena la que garantiza esa zona de rendimiento apropiado, o de fluir, sino que más bien es preciso cierto grado de respuesta somática o bien de activación física para que el deportista alcance la zona psicofísica apropiada para rendir. La autoconfianza mostró ser un factor relacionado con la sensación de balance entre destreza y reto, con la sensación de ser uno con la tarea que se está realizando y con el olvido de preocupaciones relacionadas con la propia destreza. Estas son sin duda condiciones subjetivas importantes para entrar en la zona donde se da la compenetración con la competencia, donde se logran los mejores rendimientos. En este sentido llevan razón Hernández et al., 2008) cuando señalan que la autoconfianza tiene una función mediadora fundamental en el marco de la triada que caracteriza la ansiedad precompetitiva en los deportistas (Jaenes et al., 2012).

Los datos registrados han permitido conocer sobre la autoeficacia general del grupo de jugadores y jugadoras de las selecciones nacionales de balonmano de Costa Rica y darle seguimiento a los constructos ansiedad precompetitiva y sensación de fluir a lo largo del Torneo Internacional de la Flores de Colombia. La información recopilada ha sido básica para que los entrenadores (as) de balonmano de las suprareferidas selecciones, hayan 
abordado desde una perspectiva idiográfica, la realimentación de estos deportistas en torno a la vivencia subjetiva de los mismos durante la competencia. No cabe duda que, la sistematización de la experiencia subjetiva del deportista constituye un insumo fundamental para promover el proceso metacognitivo necesario para que los (as) jugadores (as) de balonmano mejoren sus mecanismos de autoregulación emocional y afectiva.

\section{REFERENCIAS}

Abernethy, B., Maxuell, J., Masters, R., Van Der Kamp, J. \& Jackson, R. (2007). Attentional Processes in skill Learning and Expert Performance. In G. Tenenbaum \& R. Eklund (Eds.), Handbook of Sport Psychology (245-263). Hoboken, New Jersey: Wiley and Sons.

Bandura, A. (1986). Perceived self-efficacy in the exercise of control over aids infection. Evaluation and Program Planning, 13: 9-17.

Bakker, A., Oerlemans, W., Demerouti, E., Bruins, B. \& Karamat, D. (2011). Flow and performance: A study among talented Dutch soccer players. Psychology of Sport and Exercise, 12: 442-450.

Boillos, D. (2006). La mentalización en el fútbol moderno: Guía práctica para entrenadores y futbolistas. Barcelona: Biblioteca Nueva.

Breso, E., Schaufeli, W. \& Salanova, M. (2011). Can a self- efficacy based intervention decrease burnout, increase engagement and enhance performance? A quasi experimental study. High Education, 61:339-355.

Calvo, T., Jiménez, R., Santos-Rosa, J., Reina, R. \& Cervello, E. (2008). Pscychometric properties of the spanish version of the flow state scale. The Spanish Journal of Psychology, 11: 2 (660-669).

Cartoni, A.C., Mingati, C., \& Zelli , A. (2005). Gender, age, and professional level differences in the psychological correlates of fear of injury in Italian gymnastics. Journal of Sport Behavior, 28, 3-17.

Cervantes, J., Rodas, G., \& Capdevila, L. (2009). Perfil psicofisiológica de rendimiento en nadadores basado en la variabilidad de la frecuencia cardiaca y en estados de ansiedad precompetitiva. Revista de Psicología del Deporte, 18 (1), 37-52.

Ceja, L. \& Navarro, J. (2009). Dynamics of Flow: A Nonlinear Perspective. Journal Happiness Stud, 10:665-684. 
Coudevylle, G. R., Ginis, K. A. M. \& Famose, J. (2008). Determinants of selfhandicapping strategies in sport and their effects on athletic performance. Social Behaviour and Personality, 36(3): 391-398.

Covassin, T., \& Pero, S. (2004). The relationship between self-confidence, mood state, and anxiety among collegiate tennis players. Journal of Sport Behavior, 27: 230-242.

Csikszentmihalyi, M. (1992). Flow: The psychology of happiness. London: Rider.

Dosil, J. (2004). Psicología de la Actividad Física y del Deporte. Madrid: McGraw Hill.

Espada, J., González, M., Orgilés, M., Carballo, J. \& Piqueras, J. (2012). Validación de la Escala de Autoeficacia General con adolescentes Españoles. Electronic Journal of Research in Educational Psychology, 10 (1): 355-370.

Feltz, D.L., Short, S.E., \& Sullivan, P.J. (2008). Self-efficacy in sport. Champaign, IL: Human Kinetics.

Fortier, M., \& Kowal, J. (2007). The flow state and physical activity behavior change as motivational outcomes. In M. Hagger \& N. Chatzisarantis (Eds.), Intrinsic Motivation and Self Determination in Exercise and Sport (113-125). Champaign, IL: Human Kinetics.

García,T., Jiménez, R., Santos, F., Reina, R. \& Cervello, E. (2008). Psychometric Properties of the Spanish.Version of the Flow State Scale. The Spanish Journal of Psychology, 11 (2): 660-669

Grossbard, J.R., Smith, R.E., Smoll, F.L., \& Cumming, S.P. (2009). Competitive anxiety in young athletes: Differentiating somatic anxiety, worryand concentration disruption. Anxiety, Stress and Coping, 22(2), 153-166.

Hanin. Y. (2000). Emotions in Sport. Champaign, Il.: Human Kinetics.

Hanin, Y. (2007). Emotions in Sport: Current Issues and Perspectives. In G. Tenenbaum \& R. Eklund (Eds.), Handbook of Sport Psychology (31-50). Hoboken, New Jersey: Wiley and Sons.

Hein, V. \& Koka, A. (2007). Perceived Feedback and Motivation in Physical Education and Physical Activity. In M. Hagger \& N. Chatzisarantis (Eds.), Intrinsic Motivation and Self Determination in Exercise and Sport (126-140). Champaign, Il: Human Kinetics. 
Hernández, J.L., Velásquez, R., Martínez, M.E., Garoz, I. \& Tejero, C. (2011). Escala de Autoeficacia Motriz: propiedades psicométricas y resultados de su aplicación a la población escolar española. Revista de Psicología del Deporte, 20 (1): 13-28.

Hernández, R., Olmedilla, A., \& Ortega, E. (2008). Ansiedad y autoconfianza en jóvenes judokas en situaciones competitivas de alta presión. Análise Psicológica, 4 (XXVI): 689-696.

Hong, J., Chiu, P., Shih, H. \& Lin, P. (2012). Computer self-efficacy, competitive anxiety and flow state: escaping from firing online game. The Turkish Online Journal of Educational Technology, 11 (3): $70-76$.

Hodge, K., Lonsdale, C. \& Jackson, S. (2009). Athlete Engagement in Elite Sport: An Exploratory Investigation of Antecedents and Consequences. The Sport Psychologist, 2009, 23: 186-202

Jackson, S. (2000). Joy, Fun, and Flow State in Sport. En Y. Hanin (Ed.), Emotions in Sport (135-155). Champaign, Il: Human Kinetics.

Jaenes, J., Peñaloza, R., Navarrete, K. \& Bohórquez, M. (2012). Ansiedad y autoconfianza en triatletas. Revista Latinoamericana de Psicología del Ejercicio y el Deporte, 7 (1): 113-124.

Lane, A., Sewell, D., Terry, P., Bartram, D. \& Nesti, M. (1999). Confirmatory Factor Analysis of the Competitive State Anxiety Inventory - 2. Journal of Sports Sciences, 17 (6): 505-512.

León, J., Fuentes, I. \& Calvo, A. (2011). Ansiedad estado y autoconfianza precompetitiva en gimnastas. Precompetitive anxiety state and self-confidence in gymnasts. International Journal of Sport Science, 7 (23): 76-91.

Márquez, S. (2004). Ansiedad, estrés y deporte. Madrid: EOS.

Martens, R. (1987). Coaches guide to sport psychology. Champaing, IL: Human Kinetics.

Martens, R., Burton, D., Vealey, R.S., Bump, L.A., \& Smith, D.E. (1990). Development and validation of the competitive state anxiety inventory-2. En R. Martens, R.S. Vealey y D.Burton (Eds.): CompetitiveAnxiety in Sport (pp. 117-190). Champaign, IL: Human Kinetics.

Martin, J., \& Cutler, K. (2002). An exploratory study of flow and motivation in theater actors. Journal of Applied Sport Psychology, 14, 344-352. 
Mellaliu, S.D., Neil, R., \& Hanton, S. (2006). Self-confidence as a mediator of the relationship between competitive anxiety intensity and interpretation. Research Quarterly for Exercise and Sport, 77, 263-270.

Moritz, S.E., Feltz, D.L., Fahrbach, K.R. \& Mack, D.E. (2000). The relation of self efficacy measures to sport performance: A meta- analytic review: Research Quarterly for Exercise and Sport, 71, 280-294.

Myers, N. \& Feltz, D. (2007). From Self -Efficacy to Collective Efficacy in Sport: Transitional Methodological Issues. In G. Tennenbaum \& R. Eklund (Eds.), Hamdbook of Sport Psychology (pp. 799-822). Hoboken, New Jersey: Wiley \& Sons.

Nacke, L. \& Lindley, C. (2008). Flow and Immersion in First-Person Shooters: Measuring the player's gameplay experience. Future Play 2008, November 3-5.

Ramis, Y., Torregrosa, M., Viladrich, C. \& Cruz, J. (2010). Adaptación y validación de la versión española de la Escala de Ansiedad Competitiva SAS-2 para deportistas de iniciación. Psicothema, 22 (4): 1004-1009.

Ranglin, J. \& Hanin, Y. (2000). Competitive Anxiety. In Y. Hanin (Ed.), Emotions in Sport (pp. 93-112). Champaign, IL.: Human Kinetics.

Sáenz-López, P., Jiménez, F. J., Ibánez S. J., Sierra, A. y Sánchez, M. (2005). Multifactor characteristics in the process of development of the male expert basketball placer in Spain. International Journal of Sport Psychology, 36(2): 151-171.

Schüler, J. \& Engeser, S. (2009). Incentives and flow experience in learning settings and the moderating role of individual differences. In M. Wosnitza, S.A. Karabenick, A. Efklides \& P. Nenninger (Eds.), Contemporary Motivation Research: From Global to Local Perspectives. Göttingen: Hogrefe.

Seligman, M. \& Csikszentmihalyi, M. (2000). Positive Psychology. An Introduction. American Psychologist, 55 (1): 5-14.

Sherman, C. \& Poczwardowski, A. (2005). Integrating Mind and Body: Presenting Mental Skills to Young Teams. In M. Andersen (ed.), Sport pshychology in practice Champaign, IL: Human Kinetics. (pp. 17-45).

Thomas, O. Maynard, I., \& Hanton, S. (2004). Temporal Aspects of Competitive Anxiety and Self-Confidence as a Function of Anxiety Perceptions. Sport Psychologist, 18(2): 172-187

Weingerg, R. \& Gould, D. (2007). Foundations of Sport and Exercise Psychology. Champaign, IL.: Human Kinetics. 
Yamamoto, L. \& Avery, L. (2009). Optimal Experience in Sports. UCSB. Edu. Recuperado de: http://www.writing.ucsb.edu/50awards/2010_Awards/Yamamoto_Optimal_Experience_in_ Sports.pdf

Fecha de recepción: 21 de abril de 2013.

Fecha de aceptación: 4 de setiembre de 2013.

Fecha de publicación: 31 de diciembre de 2013. 